\title{
Análise Atmosférica dos Eventos de Efeito Secundário do Buraco de Ozônio Antártico Sobre o Sul do Brasil em 2012. Parte 2: Verificação Sinótica da Troposfera durante os Eventos.
}

Atmospheric Analysis of the Events Secondary Effect of Antarctic Ozone Hole Over the South of Brazil in 2012. Part 2: Synoptic verification of the troposphere during the events.

Lucas Vaz Peres ${ }^{* 1}$, Nicolle Cordero Simões dos Reis², Letícia de Oliveira dos Santos ${ }^{3}$, Gabriela Dornelles Bittencourt ${ }^{4}$, André Passaglia Schuch ${ }^{5}$, Vagner Anabor ${ }^{6}$, Damaris Kirsch Pinheiro ${ }^{7}$, Nelson Jorge Schuch ${ }^{8}$, Neusa Maria Paes Leme ${ }^{9}$

\footnotetext{
1, 6 Programa de Pós Graduação em Meteorologia, Universidade Federal Santa Maria, Santa Maria, Brasil; $2,3,4,{ }^{6}$ Graduação em Meteorologia, Universidade Federal Santa Maria, Santa Maria, Brasil;

${ }^{5}$ Programa de Pós Graduação em Biodiversidade Animal, Universidade Federal Santa Maria, Santa Maria, Brasil;

${ }^{7}$ Departamento de Engenharia Química, Universidade Federal Santa Maria, Santa Maria, Brasil;

${ }^{8}$ Centro Regional Sul de Pesquisas Espaciais - CRS/CCR/INPE-MCTI, Santa Maria, Brasil;

${ }_{9}^{9}$ INPE - Centro Regional do Nordeste - CRN/INPE-MCTI, Natal, Brasil.
}

\begin{abstract}
Resumo
No presente trabalho, foi verificada a condição sinótica da troposfera dos dois eventos de Efeito Secundário do Buraco de Ozônio Antártico ocorridos sobre o Sul do Brasil no ano de 2012. Ambos os eventos apresentaram queda média 12,1 $22,3 \%$ no conteúdo de ozônio em relação às suas médias mensais climatológica, obtidas através dos dados do Espectrofotômetro Brewer MKIII \#167 instalado no Observatório Espacial do Sul-OES/CRS/INPE-MCTI e instrumento de satélite OMI da NASA. Através de mapas de vento em 250 hPa e Omega em 500 hPa e pressão ao nível do mar e espessura entre 1000 e 500 hPa além de imagens de satélite GOES 12 do infravermelho realçado, observou-se que a condição sinótico troposférica associado com passagem de uma ampla área de estabilidade, sem nebulosidade significativa, relacionada ao afastamento da corrente de jato subtropical para o oceano atlântico, sobreposta por um amplo sistemas de alta pressão após alguns dias da passagem de um sistema frontal estacionário, favoreceu o transporte estratosférico causador de tais quedas, chegando em um dos casos a configurar condição de Bloqueio Atmosférico, sugerindo que quanto mais intensa for a massa de ar estável pós frontal, maior será a queda no conteúdo de ozônio.
\end{abstract}

Palavras-chave: Efeito Secundário do Buraco de Ozônio Antártico, Verificação Sinótica Troposférica.

\begin{abstract}
In this work, it was verified the synoptic condition in the troposphere of the two events of the Influence of the Antarctic Ozone Hole that occurred over southern Brazil in the year 2012. Both events showed average drop of the $12.1 \pm 2.3$ in the ozone content in relation to their monthly climatological averages obtained through the data of the Brewer Spectrophotometer MKIII 167 installed on the southern Space Observatory-OESCRSINPE-MCTI and instrument of OMI satellite from NASA. Through wind maps at $250 \mathrm{hPa}$ and $500 \mathrm{hPa}$ and Omega in sea level pressure and thickness between 1000 and $500 \mathrm{hPa}$ in addition to GOES 12 satellite images enhanced infrared, it was observed that the synoptic troposférica condition associated with passage of a wide area of atmospheric stability, without significant cloud cover, related to the remoteness of the subtropical jet stream to the Atlantic Ocean, superimposed by a large high-pressure systems after a few days of the passage of a frontal system stationary, favored the stratospheric transport causing such falls, arriving in one of the cases the configure Atmospheric blocking condition, suggesting that the more intense is the stable air mass front post, the greater the drop in ozone content.
\end{abstract}

Keywords: Influence of the Antarctic ozone hole, Synoptic verification in the Troposphere

* lucasvazperes@gmail.com

Recebido: 13/03/2014 Aceito: 13/03/2014 


\section{Introdução}

A partir da descoberta da massiva destruição do conteúdo de ozônio na região polar Antártica, durante as primaveras no Hemisfério Sul (Chubachi, 1984 e Farman et al. 1985), denominada de "Buraco de Ozônio Antártico" (Solomon, 1999), aumentou o interesse da comunidade cientifica no monitoramento da variabilidade do conteúdo de ozônio sobre esta região (Hofmann et al. 1997; Muller et al. 2008; Hassler et al. 2011; Salby et al. 2012), além de áreas próximas atingidas diretamente por este fenômeno, devido a passagem de sua borda sobre estas regiões, o que causa reduções do conteúdo de ozônio e consequente aumento da radiação ultravioleta que chega a superfície (Larry et al. 1995; Kirchoff et al. 1997; Perez et al. 1998 e Casiccia et al., 2008).

Regiões de médias e baixas latitudes, também podem ser influenciadas pelo Buraco de Ozônio Antártico, através do desprendimento (Prather e Jaffe,1990; Waugh, 1993) e transporte a longas distâncias, através de filamentos polares (Nash et al., 1996; Marchand et al. 2005) de massas de ar pobre em ozônio do seu interior, causando reduções temporárias na coluna total de ozônio sobre estas regiões (Waugh et al., 1994; Manney et al., 1994) .

A passagem de massas de ar estratosféricas pobre em ozônio, oriundas do Buraco de Ozônio Antártico sobre médias latitudes, foi observada primeiramente por (Kirchhoff et al., 1996) sobre o Sul do Brasil e denominada de "Efeito Secundário do Buraco de Ozônio Antártico". Este tipo de fenômeno vem sendo frequentemente observado sobre o Sul da América do Sul (Perez e Jaque, 1998; Perez et al., 2000, Paznino et al., 2008), Sul do Brasil (Pinheiro et al., 2011; 2012; Peres et al.,2011; 2012), Sul do continente africano (Semane et al., 2006; Sivakumar et al., 2007) e Nova Zelândia (Brinksma et al., 1998).

Inúmeros trabalhos científicos vêm relacionando a condição de circulação troposférica e sua influência sobre o ozônio estratosférico, sendo este tipo de estudo considerado como uma nova linha de pesquisa em ozônio (Ohring, 2010).

Distúrbios em escala sinótica na estratosfera inferior de média para altas latitudes do Hemisfério Sul são associados a sistemas sinóticos troposféricos, os quais podem pertubar a estratosfera até o nível de pressão de 50 hPa (Canziani e Legnani, 2003). A ocorrência de eventos de bloqueio atmosférico na troposfera foi associada a uma anomalia negativa no conteúdo de ozônio de 90 UD sobre a região do Conesul da América do Sul (Canziani et al., 2002).

O fluxo trans-equatorial de ozônio da região do alto Tibet em direção ao Hemisfério Sul, durante um estudo de caso em agosto de 1998, ocorreu em conjunto com a amplificação de um anticiclone no Oceano Índico no Hemisfério Sul e intensificação de um cavado no Sul da Austrália, estabelecendo uma via de transporte de ar de regiões de mínimo ozônio da estratosfera tropical, em torno das bordas de anticiclones, para regiões de máximo ozônio sobre regiões de cavados amplificados subtropicais (Rogal et al., 2010).

O principal padrão de circulação troposférico correlacionado ao transporte de ozônio estratosférico é a corrente de jato, que desempenha importante papel na distribuição vertical de ozônio (Bukin et al., 2011) e atua diretamente nas trocas entre a troposfera e a estratosfera (Tang e Prather, 2010; Manney et al., 2011a; Trickl et al., 2011).

Entretanto, pouco se sabe sobre as condições de circulação troposférica durante a ocorrência de eventos de "Efeito Secundário do Buraco de Ozônio Antártico" sobre o Sul do Brasil.

Peres et al. (2012) analisou o sistema sinótico de tempo associado ao evento de Efeito Secundário do Buraco de Ozônio Antártico ocorridos sobre o Sul do Brasil no dia 13 de outubro de 2010, sendo observada que a queda no conteúdo de ozônio associada ao transporte estratosférico da massa de ar polar pobre em ozônio, ocorreu após a passagem de um sistema frontal, onde o deslocamento da região pós frontal da corrente de jato subtropical causou intrusão de ar estratosférico para dentro da troposfera e auxiliou no transporte da massa de ar estratosférica pobre em ozônio que atingiu o Sul do Brasil.

Padrão similar de deslocamento da entrada polar da corrente de jato juntamente com o anticiclone pós frontal também foi observado no evento de Efeito Secundário do Buraco de Ozônio Antártico ocorridos sobre o Sul do Brasil no dia 14 de outubro de 2008 (Peres et al. 2011).

O presente estudo tem por objetivo verificar a condição sinótica troposférica durante dos eventos de "Efeito Secundário do Buraco de Ozônio Antártico" sobre o Sul do Brasil ocorridos no ano de 2012, a fim de identificar algum tipo de conexão entre o transporte de massas de ar pobres em ozônio na estratosfera e a condição sinótica troposfera durante a ocorrência deste tipo de evento.

\section{Metodologia}

A identificação dos eventos de "Efeito Secundário do Buraco de Ozônio Antártico" sobre o Sul do Brasil, no ano de 2012 foi mostrada na parte 1 do presente trabalho, e consistiu de uma análise de quatro principais ingredientes, de maneira análoga a realizada por Pinheiro et al., 2011; 2012; Peres 2013.

Primeiramente, foram verificadas quedas temporárias abaixo do limite estipulado de média climatológica do mês menos 1,5 do seu respectivo desvio padrão ( $\mu \mathrm{i}-1,5 \sigma \mathrm{i})$ entre os meses de agosto e novembro, nos dados da média diária da coluna total de ozônio, obtido através dos Espectrofotômetros Brewer modelo MKIV \#081 durante o período de 1992 - 2000, modelo MKII \#056 de 2000 - 2002 e modelo MKIII \#167 de 2002 até o momento, instalados no Observatório Espacial do Sul - OES/CRS/INPE - MCTI (29,4 $\left.{ }^{\circ} \mathrm{S} ; 53,8^{\circ} \mathrm{O} ; 488,7 \mathrm{~m}\right)$, em São Martinho da Serra e dos instrumentos de satélite 
TOMS e OMI da NASA, para a mesma latitude do Observatório Espacial do Sul, e em seguida realizando uma análise da dinâmica da estratosfera através de mapas de Vorticidade Potencial e vento para a superfície isentrópica de $620 \mathrm{~K}$ de temperatura potencial, trajetórias retroativas das massas de ar pobre em ozônio através do modelo Hysplit da NOAA e imagens do conteúdo de ozônio dos satélites TOMS e OMI, verificando-se a atuação do Buraco de Ozônio Antártico e sua conexão com o Sul do Brasil.

Entretanto, o foco deste trabalho é na verificação da condição sinótica troposférica durante a ocorrência de tais eventos e para isso foi realizada a análise de campos médios diários troposféricos para dois dias antes, um dia antes e no dia do evento, de variáveis meteorológicas como vento em $250 \mathrm{hPa}$ e Omega em $500 \mathrm{hPa}$ e pressão ao nível do mar e espessura entre 1000 e $500 \mathrm{hPa}$, confeccionados também com o auxílio dos parâmetros médios diários fornecidos pela National Centers for Environmental Prediction/National Atmospheric Research (NCEP/NCAR), dados de reanálise disponíveis em http://www.cdc.noaa. gov/cdc/reanalysis/reanalysis.shtml (Kalnay et al., 1996) e gerados no software de domínio público GrADS (Grid Analysis and Display System). Além disso, imagens de satélite GOES 12 do infravermelho realçado, foram utilizadas para complementar a análise da circulação troposferica.

A verificação da condição de circulação sinótica da troposfera tem por objetivo identificar os padrões sinóticos de tempo em que os eventos de "Efeito Secundário do Buraco de Ozônio Antártico" sobre o Sul do Brasil ocorreram (Peres et al., 2012), a fim de identificar alguma conexão entre a estratosfera e a troposfera durante a ocorrência deste tipo de fenômeno e verificar se a condição de circulação próxima ao nível da tropopausa pode, de alguma maneira, auxiliar no transporte das massas de ar pobre em ozônio da região do buraco de ozônio em direção ao Sul do Brasil.

\section{Resultados}

Durante o ano de 2012 foram identificados dois eventos de "Efeito Secundário do Buraco de Ozônio Antártico" sobre o Sul do Brasil, conforme apresentado na parte 1 do trabalho, onde foi realizada a análise da série de dados da coluna total de ozônio e da dinâmica da estratosfera a fim de confirmar a ocorrência dos eventos.

As datas de ocorrência dos eventos, sua respectiva média mensal climatológica com desvio padrão e porcentagem de redução na coluna de ozônio em relação à média climatológica do mês de ocorrência do evento são apresentada na tabela 1.

A seguir, será apresentada detalhadamente a verificação da condição sinótica da troposfera quando da ocorrência dos eventos de "Efeito Secundário do Buraco de Ozônio Antártico" sobre o Sul do Brasil do ano de 2012.

\begin{tabular}{cccc}
\hline $\begin{array}{c}\text { Dia do } \\
\text { Evento }\end{array}$ & $\begin{array}{c}\text { O3 } \\
\text { dia } \\
\text { (UD) }\end{array}$ & $\begin{array}{c}\text { O3 média } \\
\text { mensal (UD) }\end{array}$ & $\begin{array}{c}\text { Redução } \\
\text { O3 (\%) }\end{array}$ \\
\hline $\mathbf{1 4 / 0 9 / 2 0 1 2}$ & 267,8 & $298,87 \pm 9,80$ & $10,4 \%$ \\
\hline $\mathbf{1 4 / 1 0 / 2 0 1 2}$ & 252,6 & $292,73 \pm 9,85$ & $13,7 \%$ \\
\hline
\end{tabular}

Tabela 1: Datas de ocorrência dos eventos, sua respectiva média mensal climatológica com desvio padrão e porcentagem de redução na coluna de ozônio em relação à média climatológica do mês de ocorrência dos eventos de "Efeito Secundário do Buraco de Ozônio Antártico" sobre o Sul do Brasil no ano de 2012.

Os platôs de figuras utilizados para essa verificação apresentam em suas linhas, de cima para baixo, os campos de vento em $250 \mathrm{hPa}$ e Omega em $500 \mathrm{hPa}$, pressão ao nível do mar e espessura entre 1000 e $500 \mathrm{hPa}$ e a imagens de satélite GOES 12 do infravermelho realçado para os dias representados nas colunas, sendo a primeira dois dias antes, a segunda um dia antes e a terceira o dia do evento em si, mostrando a evolução temporal das variáveis meteorológicas em questão.

\subsection{Evento de 14 de setembro de 2012}

A verificação da condição de tempo troposferica, apresentada na Figura 1, para o evento "Efeito Secundário do Buraco de Ozônio Antártico" sobre o Sul mostra que sobre esta região, atuava uma ampla área de estabilidade atmosférica, a partir do afastamento para o oceano Atlântico de áreas de instabilidade no dia 12 de setembro até o dia 14 de setembro, dia da maior queda no conteúdo de ozônio para este evento.

No dia 12 de setembro, a alta troposfera foi analisada através do campo de vento em 250 hPa e Velocidade Vertical Omega em $500 \mathrm{hPa}(\mathrm{A})$, sendo observado a atuação de um pequeno núcleo da corrente de jato subtropical, resquício do sistema frontal que atuou de forma estacionária sobre o Sul do Brasil desde o dia 09 ate o dia 12 de setembro, com um núcleo de valores negativos de velocidade vertical Omega, centrado sobre o Estado do Rio Grande do Sul, que em conjunto com o cavado invertido no campo de pressão ao nível do mar (D), que se estendeu desde o Estado do Mato Grosso do Sul, passando pelo sul do Paraguai, Paraná, Santa Catarina e Norte do Rio Grande do Sul, favoreceu a formação de nebulosidade pouco intensa observada na imagen de satélite GOES 12 do infravermelho realçado para as 18 UTC (G) deste mesmo dia.

Ainda no dia 12, observa-se a presença de um núcleo de valores positivos de velocidade vertical Omega a sul da foz do Rio do Prata, posicionada sobre a região de entrada polar da corrente de jato subtropical, provável posição da massa de ar pobre em ozônio na estratosfera.

A partir do dia 13 de setembro, o pequeno núcleo 

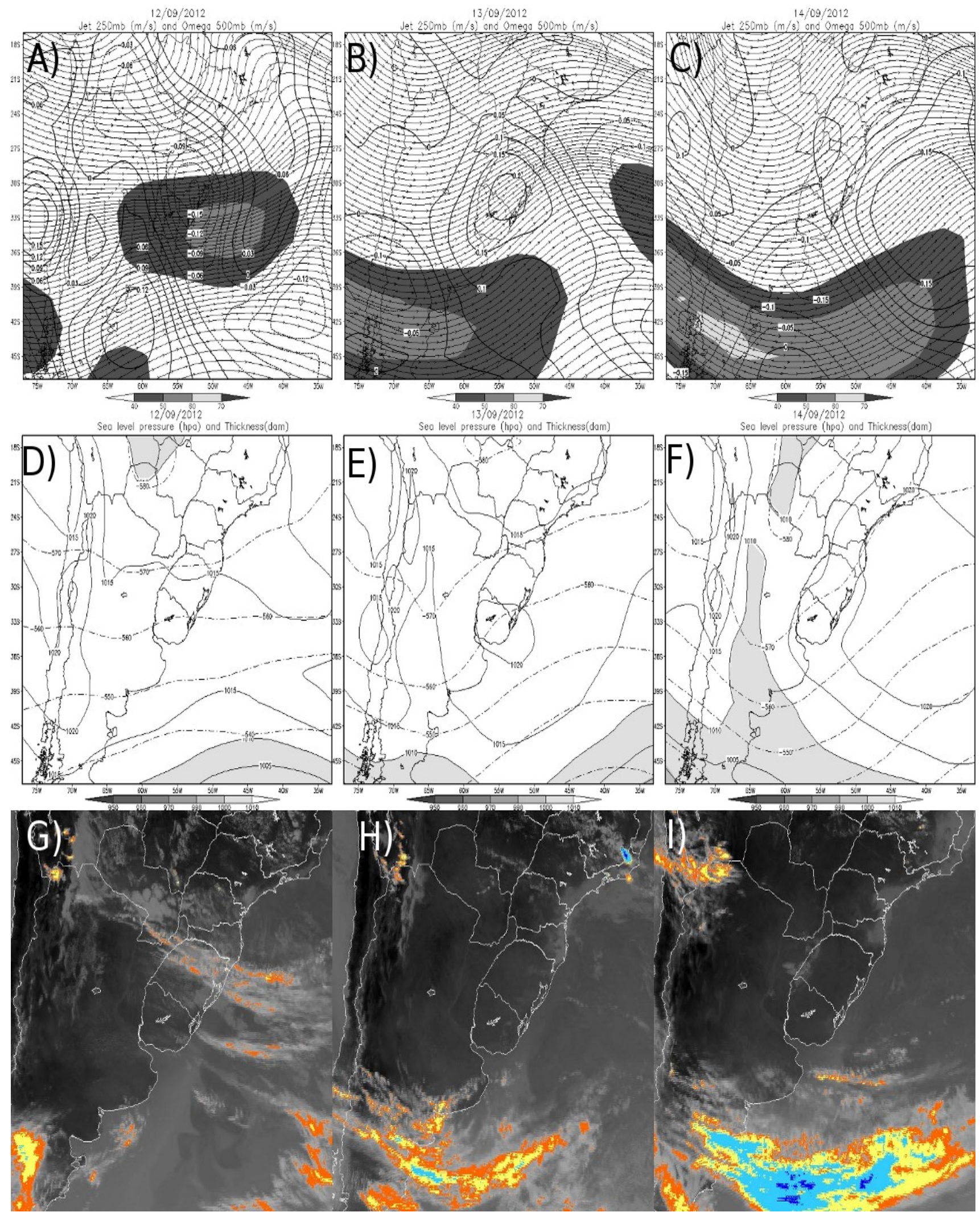

Figura 1- Campos médios diários de vento em 250 hPa e Omega em 500 hPa (A, B e C), Pressão ao nível do mar e espessura entre 1000 e $500 \mathrm{hPa}(\mathrm{D}, \mathrm{E}$ e F), e imagens do satélite GOES 12 do infravermelho realçado para as 18:00 UTC (G, H e I) para os dias 12, 13 e 14 de setembro de 2012 respectivamente. 
da corrente de jato subtropical se desloca em direção ao oceano Atlântico, permitindo o deslocamento sobre o Sul do Brasil de uma amplo centro de valores positivos de velocidade vertical Omega no campo de vento em 250 hPa e Velocidade Vertical Omega em 500 hPa (b), que no dia anterior atuava sobre o Centro Sul da Argentina sobre a região de entrada polar da corrente de jato subtropical, característico por movimento subsidente e intrusão de ar estratosférico para dentro da troposfera.

Ao Sul da província de Buenos Aires observa-se a chegada pelo Oceano Pacífico da corrente de jato polar, a qual, ao movimentar-se de Sul para norte, seu setor de saída equatorial, auxilia mais ainda no deslocamento da região de valores positivos de velocidade vertical Omega, por sobre a região Sul do Brasil.

Este sistema na alta troposfera, associado com o deslocamento sobre o Sul do Brasil do anticiclone migratório pós-frontal observado no campo de pressão ao nível do mar e espessura entre os níveis de 1000 e 500 $\mathrm{hPa}(\mathrm{E})$, característico por divergência do ar em baixos níveis foram responsáveis pela atuação de uma massa de ar estável sem nebulosidade significativa observada na imagem do infravermelho realçada do satélite GOES 12 para 18 UTC da presente data $(\mathrm{H})$.

O amplo núcleo de valores positivos de velocidade vertical Omega, que estava a Sul da foz do Rio do Prata se deslocou para norte por sobre o Uruguai, Nordeste da Argentina, Sul do Paraguai e Estados Brasileiros do Rio Grande Do Sul, Santa Catarina e Paraná, que acoplada ao transporte das massas de ar na estratosfera oriunda da região polar, ocasionou uma queda no conteúdo de ozônio sobre o Sul do Brasil de 6,1\%, sendo a segunda maior queda para este evento, perdendo apenas para o dia 14, observado na Figura 1 da Parte 1 da respectiva pesquisa .

Dia 14 de setembro, dia da maior queda no conteúdo de ozônio observado para o evento, a corrente de jato polar prossegue atuando a Sul da província de Buenos Aires, porém mais deslocadas sobre o Oceano Atlântico, mantendo as condições favoráveis para a manutenção da atuação da massa de ar estável, devido à permanência da atuação do amplo centro de valores positivos de velocidade vertical Omega, sobre a região Sul do Brasil (C), mesmo que deslocado sobre o oceano Atlântico.

Associado a situação descrita para a alta troposfera, o anticiclone de elevados valores no campo de pressão ao nível do mar, baixa troposfera, centrado sobre o Oceano Atlântico na direção da costa do Uruguai e estado do Rio Grande do Sul (F), prossegue favorecendo a atuação da ampla área de estabilidade do ar, inibindo a formação de nebulosidade significativa sobre a região Sul do Brasil, observada na imagem do infravermelho realçado do satélite GOES 12 para 18 UTC para o respectivo dia (I).

Ainda no dia 14, sobre a região da foz do Rio da Prata, observa-se o posicionamento de um cavado no campo de vento com valores negativos de velocidade vertical Omega em seu interior no escoamento na alta troposfera, associado com a advecção de calor e umidade da região amazônica, sobre as regiões de baixa pressão continental costeando as cordilheiras dos Andes em direção a região centro Sul da Argentina, favorecem a formação de nebulosidade significativa sobre esta região, ficando o Sul do Brasil em uma situação pré frontal ao longo de alguns dias, podendo em a massa de ar estável manter por vários dias, configurar condição de Bloqueio Atmosférico (Kayano e Kousky, 1990), o que não foi confirmado para este evento por ter, a massa de ar estável, atuado por apenas 3 dias.

Entretanto, este padrão de circulação atmosférica, com o deslocamento da corrente de jato subtropical de médias para baixas latitudes, posicionando sua região entrada polar, com um amplo núcleo de valores positivos de velocidade vertical Omega, auxiliada pelo deslocamento da região de saída equatorial da corrente de jato polar que atua sobre o centro sul da Argentina na alta troposfera.

Somado ao posicionamento do anticiclone migratório após a passagem de uma frente fria estacionária dias antes da maior queda no conteúdo de ozônio, podem ter auxiliado, na ocorrência de intrusão de ar estratosférico para dentro da troposfera e no transporte horizontal da massa de ar estratosférica pobre em ozônio da região Antártica em direção ao Sul do Brasil.

Esta configuração demostra uma clara evidência de conexão entre o transporte de massas de ar na estratosfera e o deslocamento de sistemas sinóticos na troposfera, com a passagem de um sistema frontal estacionário e estabelecimento de uma massa de ar estável, perdurando por poucos dias, antes da chegada de um novo sistema frontal como o observado para este estudo de caso.

\subsection{Evento de 14 de outubro de 2012}

Verificando a condição de tempo troposferica, para o evento de Efeito Secundário do Buraco de Ozônio Antártico sobre o Sul do Brasil do dia 14 de outubro de 2012, apresentada na Figura 2, mostra que sobre esta região, passava uma ampla área de estabilidade atmosférica, precedida pela passagem de um sistema frontal estacionário até o dia 10 de outubro e seguida por áreas de instabilidade relacionadas a diminuição da pressão sobre o continente ocasionada pela advecção de calor e temperatura da Região Amazônica a partir do dia 16 de outubro.

Acompanhando a passagem desta massa de ar estável na troposfera, massas de ar pobre em ozônio na estratosfera se desprenderam da região do Buraco de Ozônio na Antártica e atingiram o sul do Brasil, e causar uma queda de $13,7 \%$ no conteúdo de ozônio do dia 14 de outubro, dia da maior queda no conteúdo de ozônio para este evento.

Analisando o dia 12 de outubro, observa-se a alta troposfera através do campo de vento em $250 \mathrm{hPa}$ e Velocidade Vertical Omega em $500 \mathrm{hPa}(\mathrm{A})$, verificando 
$2 / 10 / 2012$


Figura 2- Campos médios diários de vento em 250 hPa e Omega em 500 hPa (A, B e C), Pressão ao nível do mar e espessura entre 1000 e $500 \mathrm{hPa}(\mathrm{D}, \mathrm{E}$ e F), e imagens do satélite GOES 12 do infravermelho realçado para as 18:00 UTC (G, H e I) para os dias 12, 13 e 14 de outubro de 2012 respectivamente. 
a atuação de um pequeno núcleo da corrente de jato subtropical, sobre a região Sudeste do Brasil, resquício do lento deslocamento do sistema frontal estacionário que passou de forma estacionária até o dia 10 de outubro sobre o Sul do Brasil, deixando esta região, no dia 12, sobre a atuação de uma amplo núcleo de valores positivos de velocidade vertical Omega, que se estende do Norte do Paraguai, centro Norte da Argentina, passando pelo Uruguai e Estado do Rio Grande do Sul, até grande distancia do litoral adjacente.

Na baixa troposfera, nota-se a presença do anticiclone migratório pós-frontal, centrado sobre o oceano atlântico adjacente a foz do Rio da Prata no campo de pressão ao nível do mar (D), que em conjunto com o amplo núcleo de valores positivos de velocidade vertical Omega na alta troposfera, favoreceram o estabelecimento de uma ampla área de estabilidade atmosférica sobre o Sul do Brasil, que inibiu a formação de nebulosidade, observado na imagem do satélite GOES 12 do infravermelho realçado para as $18 \mathrm{UTC}(\mathrm{G})$ do dia 12 de outubro. Esta condição sinótica caracteriza um sistema de dipolo, onde o Sul do Brasil se encontra numa condição de estabilidade atmosférica e o centro Sul da Argentina e a região Sudeste do Brasil se encontram em uma condição de instabilidade atmosférica.

No dia 13 de outubro, este sistema de dipolo na alta troposfera, observada nos valores de velocidade vertical Omega no campo de vento em $250 \mathrm{hPa}$ e Velocidade Vertical Omega em 500 hPa (b), com condição de estabilidade atmosférica sobre o Sul do Brasil (núcleo de valores positivos) e instabilidade atmosférica sobre o centro Sul da Argentina e a região Sudeste do Brasil (núcleos de valores negativos) apenas se desloca lentamente para norte, mantendo a atuação do amplo núcleo de valores positivos de velocidade vertical Omega desde o Norte do Paraguai, centro Norte da Argentina, passando pelo Uruguai e Estado do Rio Grande do Sul, e litoral adjacente.

Juntamente com este sistema na alta troposfera, o anticiclone migratório pós-frontal observado no campo de pressão ao nível do mar e espessura entre os níveis de 1000 e $500 \mathrm{hPa}$ (E), também se desloca lentamente para norte e Oceano Atlântico na baixa troposfera, mantendo a atuação da massa de ar estável sem nebulosidade significativa observada na imagem do infravermelho realçada do satélite GOES 12 para 18 UTC do dia 13 de outubro $(\mathrm{H})$.

No dia 14 de outubro, dia da maior queda no conteúdo de ozônio para o evento, prossegue o lento deslocamento do amplo núcleo de valores positivos de velocidade vertical Omega, favorecendo a manutenção da atuação da massa de ar estável, sobre a região Sul do Brasil (C) e a chegada sobre a foz do Rio da Prata e Uruguai da borda do núcleo de valores negativos de velocidade vertical Omega na alta troposfera.

Na baixa troposfera, o anticiclone no campo de pressão ao nível do mar (F), também se desloca para norte e leste, mantendo a atuação sobre o Sul do Brasil da ampla área de estabilidade atmosférica, que favorece a inibição de formação de nebulosidade sobre a região Sul do Brasil, observada na imagem do infravermelho realçado do satélite GOES 12 para 18 UTC do dia 14 de outubro (I).

É observada também no dia 14, a formação de uma ampla área de baixa pressão continental costeando as Cordilheiras dos Andes desde o Norte do Paraguai até o Centro Sul da Argentina na borda do anticiclone que atua sobre o Sul do Brasil, advectando calor e umidade da região amazônica, sobre estas regiões.

Entretanto, como a massa de ar estável atuou por seis dias sobre o Sul do Brasil, do dia 11 a 16 de outubro (restante da análise para os dias fora de entre 12 e 14, não mostrada), pode-se caracterizar este evento como um Bloqueio Atmosférico na troposfera do tipo dipolo (Van Loon (1956), constituído de um anticiclone de grande amplitude e um ciclone em latitudes mais baixas, a norte deste, sendo frequente a observação da ocorrência deste tipo de evento sobre o Sul do Brasil (Marques, 1996, Nascimento e Ambrizzi (2002)), como observado para ente estudo de caso, através da evolução temporal dos campos de pressão ao nível do mar e espessura entre os níveis de 1000 e 500 hPa (D,E e F).

O padrão de circulação troposférica, com a passagem de um sistema frontal estacionário dias antes da ocorrência da maior queda na coluna de ozônio sobre Sul do Brasil, e estabelecimento de uma ampla área de estabilidade atmosférica pós frente estacionária, atuando por diversos dias caracterizando um sistema de Bloqueio Atmosférico, favoreceram o transporte da massa de ar estratosférica pobre em ozônio da região Antártica em direção ao Sul do Brasil, evidenciando a existência de conexão entre a passagem de um sistema sinótico estacionário na troposfera como o Bloqueio Atmosférico e o transporte de massas de ar na estratosfera como ocorrido para este evento do dia 14 de outubro de 2012.

\subsection{Discussões}

O Sul do Brasil tem por característica a passagem de distúrbios troposféricos transientes, sendo atingida por diversos fenômenos meteorológicos de tempo em escala sinótica. Os mais significativos na baixa troposfera são a passagem de frentes frias, formação de sistemas convectivos de mesoescala e ciclones extratropicais, já na alta troposfera, a formação e passagem das correntes de jatos subtropical e polar e bloqueios atmosféricos (Satyamurty et al, 1998, Reboita et al., 2008).

Em virtude disso, é esperado que a chegada de massas de ar estratosféricas pobre em ozônio sobre o Sul do Brasil, coincida com a ocorrência de algum destes fenômenos mencionados. Como a previsão de tempo troposférica ocorre diariamente de forma operacional, torna-se importante compreender a conexão entre o sistema sinótico troposférico de tempo e o transporte de massas de ar pobre em ozônio oriundas do Buraco de 
Ozônio Antártico que atingem o Sul do Brasil, de forma a facilitar futuras identificações e previsão de ocorrência deste tipo de fenômeno.

Sobre o Sul do Brasil este tipo de fenômeno vem sendo constantemente observado, causando queda temporária média na coluna total de ozônio Pinheiro et al., 2011, 2012), comprovando a importância do tema devido a reduções de $1 \%$ no conteúdo de ozônio causarem aumento médio de 1,2\% na Radiação Ultravioleta sobre esta região (Guarnieri et al., 2004). Além disso, costumam estar associados a condições de tempo sinóticas na troposfera, principalmente relacionadas a condições pós frontais de massas de ar estáveis (Peres et al,. 2011, 2012).

O evento de Efeito Secundário do Buraco de Ozônio Antártico sobre o Sul do Brasil do dia 14 de setembro de 2012 na estratosfera ocorreu após a passagem de uma frente fria estacionária dias antes da ocorrência do evento e atuação durante três dias de uma massa de ar estável pós-frontal antes da passagem de um próximo sistema frontal.

Esta condição sinótica da troposfera é muito semelhante às observadas por Peres et al. (2011 e 2012), onde a corrente de jato, principal padrão de circulação troposférica correlacionado ao transporte de ozônio na estratosfera, (Bukin et al., 2011) se desloca sobre o Sul do Brasil em direção a região Sudeste, trazendo a sua retaguarda a região de entrada polar característica por movimento subsidente e núcleo de valores positivos de velocidade vertical Omega, que favorecem a instalação e manutenção da massa de ar estável sem nebulosidade significativa observada durante os dias de queda no conteúdo de ozônio observados neste evento.

Entretanto, o evento de Efeito Secundário do Buraco de Ozônio Antártico sobre o Sul do Brasil do dia 14 de outubro de 2012 na estratosfera, também ocorreu após a passagem de uma frente fria estacionária dias antes da ocorrência do evento, porém a massa de ar estável pós frontal atuou durante seis dias antes da passagem do próximo sistema frontal, caracterizando uma condição de Bloqueio Atmosférico, condição troposférica esta, em que Canziani et al. (2002) associou a anomalias negativas no conteúdo de ozônio sobre a região do Cone Sul da América do Sul.

Torna-se importante a verificação da intensidade durante sua passagem, da massa de ar estável pós frontal na troposfera, fator relevante na intensidade da queda no conteúdo de ozônio relacionada ao transporte das massas de ar de origem polar na estratosfera, como o observado no ano de 2012, com a maior queda no conteúdo de ozônio, para o dia 14 de outubro, ocorrendo juntamente a massa de ar estável que atuou por mais tempo na troposfera antes da passagem do próximo sistema frontal, sugerindo que quanto mais intensa for a massa de ar estável pós frontal, maior será a queda no conteúdo de ozônio estratosférico.

\section{Conclusões}

Na parte 1 do respectivo estudo, identificou-se a ocorrência de dois eventos de Efeito Secundário do Buraco de Ozônio Antártico sobre o Sul do Brasil no ano de 2012, um no dia 14 de setembro, apresentando uma queda de 10,4\% no conteúdo de ozônio em relação à média mensal climatológica do mês de setembro e outro no dia 14 de outubro, apresentando uma queda de $13,7 \%$ no conteúdo de ozônio em relação à média mensal climatológica do mês de outubro, ambos confirmados através da análise da dinâmica da estratosfera para os eventos em questão.

Verificando-se a condição sinótica da troposfera nos dois eventos de Efeito Secundário do Buraco de Ozônio Antártico sobre o Sul do Brasil no ano de 2012, observouse que o transporte da massa de ar estratosférica pobre em ozônio que atingiu o Sul do Brasil no evento do dia 14 de setembro, ocorreu em conjunto com atuação de uma ampla área de estabilidade atmosférica ocorrida após a passagem de um sistema frontal estacionário na troposfera, sem nebulosidade significativa, ocasionada pelo afastamento para o oceano Atlântico da região de entrada polar da corrente de jato subtropical em conjunto com a atuação de um sistema de alta pressão pós frontal, caracterizados por causar subsidência das massas de ar, inibindo assim a formação de nebulosidade.

Durante o evento de Efeito Secundário do Buraco de Ozônio Antártico sobre o Sul do dia 14 de outubro de 2012, a condição sinótica da troposféra verificada foi semelhante a do evento anterior, também ocorrendo após a passagem de uma sistema frontal estacionário dias antes da maior queda no conteúdo de ozônio, porém neste caso, amassa de ar estável pós frontal atuou durante período de tempo suficiente para caracterizar uma condição de Bloqueio Atmosférico, já associada a ocorrência de anomalias negativas no conteúdo de ozônio.

Resalta-se a importante da verificação para compreender melhor a conexão entre o sistema sinótico troposférico de tempo e o transporte de massas de ar pobre em ozônio oriundas do Buraco de Ozônio Antártico que atingem o Sul do Brasil, de forma a facilitar futuras identificações e possibilidade de previsão de ocorrência deste tipo de fenômeno.

Torna-se importante a verificação da intensidade da massa de ar estável pós frontal na troposfera e a intensidade da queda no conteúdo de ozônio relacionada ao transporte das massas de ar de origem polar na estratosfera, uma vez que o evento de maior queda no conteúdo de ozônio ocorreu junto a massa de ar estável que atuou por mais dias, sugerindo que quanto mais intensa for a massa de ar estável pós frontal, maior será a queda no conteúdo de ozônio estratosférico.

A influência da diminuição do conteúdo de ozônio sobre a radiação ultravioleta para os eventos de Efeito Secundário do Buraco de Ozônio Antártico sobre o Sul do Brasil no ano de 2012 serão assunto da terceira e ultima parte desta pesquisa. 


\section{Agradecimentos}

Este trabalho integra o Programa de Pós-Graduação em Meteorologia da Universidade Federal de Santa Maria (UFSM) juntamente com o Instituto Nacional de Ciência e Tecnologia Antártico de Pesquisas Ambientais (INCT -APA), que recebe apoio científico e financeiro do Conselho Nacional de Pesquisa e Desenvolvimento (CNPq process: n 574018/2008-5) e Fundação Carlos Chagas de Amparo à Pesquisa do Estado do Rio de Janeiro (FAPERJ $n^{\circ}$ E-16/170.023/2008). Os autores também agradecem o apoio dos Ministérios da Ciência, Tecnologia e Inovação (MCTI), do Ministério do Meio Ambiente (MMA) e da Comissão Interministerial da para os Recursos do Mar (CIRM). Agradecimentos também para FAPEREGS / CAPES pela Bolsa de estudo, a NASA / TOMS e NCEP / NCAR pelos dados, e NOAA pelo modelo HYSPLIT utilizados nas análises e ao Projeto ATMANTAR, edital MCTI/CNPq do Ano Polar Internacional, processo $\mathrm{n}^{\circ}$ .52.0182/2006-5.

\section{Referências}

BRINKSMA, E. J.; MEIJER, Y. J.; CONNOR, B. J.; MANNEY, G. L.; BERGWERFF, J. B.; BODEKER, G. E.; BOYD, I. S.; LILEY, J. B.; HOGERVORST, W.; HOVENIER, J. W.; LIVESEY, N. J.; SWART, D. P. $\mathrm{J}$. Analysis of record-low ozone values during the 1997 winter over Lauder, New Zealand. Geophys. Res. Lett., v. 25, n. 15, p. 2785-2788, 1998.

BUKIN, O. A., SUAN An, N.; PAVLOV, A. N.; STOLYARCHUK, S. Y. and SHMIRKO, K .A. Effect that Jet Streams Have on the Vertical Ozone Distribution and Characteristics of Tropopause Inversion Layer, Izvestiya Atmospheric and Oceanic Physics, v. 47, n. 5, p. 610-618, 2011.

CANZIANI, P. O.; COMPAGNUCCI, R. H.; BISCHOFF, A. S. AND LEGNANI, W. E. A study of impacts of tropospheric synoptic processes on the genesis and evolution of extreme total ozone anomalies over southern South America. JOURNAL OF GEOPHYSICAL RESEARCHATMOSPHERES. v. 107, n. D24, 4741, doi: 10.1029/2001JD000965, 2002.

CANZIANI, P. O. AND LEGNANI, W. E. Tropospheric-stratospheric coupling: Extratropical synoptic systems in the lower stratosphere. QUARTERLY JOURNAL OF THE ROYAL METEOROLOGICAL SOCIETY. v. 129, n. 592, p. 2315-2329, doi: 10.1256/qj.01.109, Part a, 2003.
CASICCIA, C.; ZAMORANO, F.; HERNANDEZ, A. Erythemal irradiance at the Magellan's region and Antarctic ozone hole 1999-2005. Atmosfera, v. 21 n.1, p. 1-12, 2008.

CHUBACHI, S. Preliminary result of ozone observations at Syowa Station from February, 1982 to January, 1983. Mem. Natl. Inst. Polar Res. Jpn. Spec., v. 34, p. 13-20, 1984.

FARMAN, J. C.; GARDINER, B. G.; SHANKLIN, J. D. Large losses of total ozone in Antarctica reveal seasonal ClOx/NOx interaction. Nature. 315: 207210, 1985.

GUARNIERI, R. A.; PADILHA, L. F.; GUARNIERI, F. L.; ECHER, E.; MAKITA, K.; PINHEIRO, D. K.; SCHUCH, A.M.P.; BOEIRA, L. S.; SCHUCH, N.J. A study of the anticorrelations between ozone and UV-B radiation using linear and exponential fits in southern Brazil. Adv. Space Res., v. 34, p. 764-768, 2004.

HASSLER, B.; DANIEL, J. S.; JOHNSON, B. J.; SOLOMON, S. AND OLTMANS, S. J. An assessment of changing ozone loss rates at South Pole: Twenty-five years of ozonesonde measurements. JOURNAL OF GEOPHYSICAL RESEARCH-ATMOSPHERES. v: 116, D22301, doi: 10.1029/2011JD016353, 2011.

HOFMANN, D. J.; OLTMANS, S. J.; HARRIS, J. M.; JOHNSON, B. J. and LATHROP, J. A. Ten years of ozone sonde measurements at the South Pole: Implications for recovery of springtime Antarctic ozone. J. Geophys. Res., v.102, p.8931-8943, 1997.

KALNAY, E.; KANAMITSU, M.; KISTLER, R.; COLLINS, W.; DEAVEN, D.; GANDIN, L.; IREDELL, M.; SAHA, S.; WHITE, G.; WOOLLEN, J.; ZHU, Y.; CHELLIAH, M.; EBISUZAKI, W.; HIGGINS, W.; JANOWIAK, J.; MO, K. C.; ROPELEWSKI, C.; WANG, J.; LEETMAA, A.; REYNOLDS, R.; JENNE, R.; JOSEPH, D. The NCEP/ NCAR 40-year reanalysis project. Bull. Amer. Met. Soc., v. 77, n. 3, p. $437-471,1996$.

KIRCHHOFF, V. W. J. H.; SCHUCH, N. J.; PINHEIRO, D. K.; HARRIS, J. M. Evidence for an ozone hole perturbation at $30^{\circ}$ south. Atmos. Environ., v. 33, n. 9, p. 1481-1488, 1996.

KIRCHHOFF, V. W. J. H.; SAHAI, Y.; CASICCIA, C. A. R.; ZAMORANO, F.; VALDERRAMA, V. Observations of the 1995 ozone hole over Punta Arenas, Chile. J. Geophys. Res-Atmos., v. 102, n. D13, p. 16109-16120, 1997. 
KAYANO, M. T.; KOUSKY, V. E. Southern Hemisphere blocking: a comporison between two indices. Meteorology and Atmospheric Physics, v.42, p. 165-170,1990.

LARY, D.; CHIPPERFILD, M.; PYLE, J.; NORTON, W.; RIISHOJGAARD. L. Tree-dimensional tracer initialization and general diagnostics using equivalent PV latitude-potential-temperature coordinates, Q. J. Roy. Metror. Soc., v. 121, p. 187210, 1995.

MANNEY, G. L.; ZUREK, R. W.; NEIL, A. O.; SWINBANK, R. On the motion of air through the stratospheric polar vortex. J. Atmos. Sci., v. 51, p. 2973-2994, 1994.

MANNEY, G. L.; HEGGLIN, M. I.; DAFFER, W. H.; SANTEE, M. L.; RAY, E. A.; PAWSON, S.; SCHWARTZ, M. J.; BOONE, C. D.; FROIDEVAUX, L.; LIVESEY, N. J.; READ, W. G.; WALKER, K. A. Jet characterization in the upper troposphere/lower stratosphere (UTLS): applications to climatology and transport studies. Atmos. Chem. Phys., v. 11, p. 6115-6137, 2011a.

MARCHAND, M.; BEKKI, S.; PAZMINO, A.; LEFÈVRE, F.; GODIN-BEEKMANN, S.; HAUCHECORNE, A. Model simulations of the impact of the 2002 Antarctic ozone hole on midlatitudes. J. Atmos. Sci., v. 62, p. 871-884, 2005.

MÜLLER, R., GROOß, J. -U., LEMMEN C., HEINZE, D., DAMERIS, M. AND BODEKER G. Simple measures of ozone depletion in the polar stratosphere. Atmos. Chem. Phys. v. 8, p. 251-264, 2008.

NASH, E. R.; NEWMAN, P. A.; ROSENfiELD, J. E.; SCHOEBERL, M. E. An objective determination of the polar vortex using Ertel's potential vorticity. J. Geophys. Res., v. 101, p. 9471-9478, 1996.

OHRING, G.; BOJKOV, R. D.; BOLLE, H. J, HUDSON, R. D. and VOLKERT, H. Radiation and Ozone: Catalysts for Advancing International Atmosferic Science Programmes for over half a century. Space research Today, n177, April 2010.

PAZMINO, A. F.; GODIN-BEEKMANN, S.; LUCCINI, E. A.; PIACENTINI, R. D.; QUEL, E. J.; HAUCHECORNE, A. Increased UV radiation due to polar ozone chemical depletion and vortex occurrences at Southern Sub-polar Latitudes in the period [1997-2005]. Atmos. Chem. Phys., v. 8, n. 17, p. 5339-5352, 2008.
MARQUES, R. F. C. Bloqueio atmosférico no Hemisfério Sul. São José dosCampos, SP. 1996. (INPE-6742-TDI/632). Tese (Doutorado em Meteorologia) -Instituto Nacional de Pesquisas Espaciais, São José dos Campos, SP, 1996.

NASCIMENTO, E. L.; AMBRIZZI T. The influence of atmospheric blocking on the Rossby wave propagation in Southern Hemisphere winter flows. Journal of the Meteorological Society of Japan, v. 80, p. 139-159, 2002.

PERES, L. V.; KALL, E.; CRESPO, N.M; FONTINELE, J. L.; ANABOR, V.; PINHEIRO, D. K.; SCHUCH, N.J.; LEME, N.M.P. Caracterização sinótica do evento de Efeito Secundário do Buraco de Ozônio Antártico sobre o Sul do Brasil do dia 14/10/2008. Ciência e Natura, Ed. Suplementar, Universidade Federal de Santa Maria, p. 323 - 326, 2011.

PERES, L. V.; Crespo, N., M.; Da Silva, O.; K., HUPFER, N.; Anabor, V.; PINHEIRO, D. K.; SCHUCH, N. J.; LEME, N., P. Sinoptic weather system associate with influence of the Antartic Ozone Hole over South of Brazil at October, 13th, 2010. Annual Active Report 2011 - National Institute of Science and Technology Antarctic Environmental Research., v. 1, p. 30-33, 2012.

PERES, L. V. Efeito Secundário do Buraco de Ozônio Antártico Sobre o Sul do Brasil. Dissertação (Mestrado em Meteorologia). Universidade Federal de Santa Maria, 2013.

PEREZ, A.; DE CARCER, I. A.; TOCHO, J.O.; CRINO, E.; SANDOVAL, H. F. R.; BERNI, M. E.; DA SILVA, L.; HENRIQUES, D.; CUSSO, F.; JAQUE, F. The extent of the ozone hole over South America during the spring of 1993, 1994, and 1995. J. Phys. D Appl. Phys., v. 31, n. 7, p. 812-819, 1998.

PEREZ, A.; JAQUE, F. On the Antarctic origin of low ozone events at the South American continent during the springs of 1993 and 1994. Atmos. Environ., v. 32, n. 21, p. 3665-3668, 1998.

PEREZ, A.; CRINO, E.; DE CARCER, I. A.; JAQUE, F. Low-ozone events and three-dimensional transport at midlatitudes of South America during springs of 1996 and 1997. J. Geophys. Res-Atmos., v. 105, n. D4, p. 4553-4561, 2000.

PINHEIRO, D. K.; LEME, N. P.; PERES, L. V.; KALL, E. Influence of the Antarctic ozone hole over South of Brazil in 2008 and 2009. Annual Active Report 2010 - National Institute of Science and Technology Antarctic Environmental Research, v. 1, p. 33-37, 2011. 
PINHEIRO, D. K.; PERES, L. V.; Crespo, N. M.; SCHUCH, N. J.; LEME, N., P. Influence of the Antarctic ozone hole over South of Brazil in 2010 and 2011. Annual Active Report 2011 - National Institute of Science and Technology Antarctic Environmental Research, v. 1, p. 34-38, 2012.

PRATHER, M.; JAFFE, H. Global impact of the Antarctic ozone hole: chemical propagation. J. Geophys. Res-Atmos., v. 95, p. 3413-3492, 1990.

REBOITA, M. S. Ciclones Extratropicais sobre o Atlântico Sul: Simulação Climática e Experimentos e Sensibilidade. Tese de Doutorado em Meteorologia, Instituto de Astronomia, Geofísica e Ciências Atmosféricas - IAG-USP, 359 pg., 2008.

ROGAL, M.; HITCHMAN, M. H.; BUKER, M. L..; TRIPOLI, G. J.; STAJNER, I. AND HAYASHI, $\mathrm{H}$. Modeling the effects of Southeast Asian monsoon outflow on subtropical anticyclones and midlatitude ozone over the Southern Indian Ocean. JOURNAL OF GEOPHYSICAL RESEARCH-ATMOSPHERES. v. 115, D20101, doi: 10.1029/2009JD012979, 2010.

\section{SALBY, M. L.; TITOVA, E. A. AND DESCHAMPS,} L. Changes of the Antarctic ozone hole: Controlling mechanisms, seasonal predictability, and evolution. JOURNAL OF GEOPHYSICAL RESEARCH-ATMOSPHERES. v. 117, D10111, doi: 10.1029/2011JD016285, 2012.

SATYAMURTY, P.; NOBRE, C. A.; SILVA DIAS, P. L. South America. In: Meteorology of the Southern Hemisphere. Eds. Karoly D. J.; Vicent, D. G. American Meteorological Society, Meteorological Monographs, v. 27, n. 49, p. 119-139, 1998.

SEMANE, N.; BENCHERIF, H.; MOREL, B.; HAUCHECORNE, A.; DIAB, R. D. An unusual stratospheric ozone decrease in Southern Hemisphere subtropics linked to isentropic airmass transport as observed over Irene (25.5 $S, 28.1^{\circ}$ E) in mid-May 2002. Atmos. Chem. Phys., v. 6, p. 1927-1936, 2006.

SIVAKUMAR, V.; PORTAFAIX, T.; BENCHERIF, H.; GODIN-BEEKMANN, S.; BALDY, S. Stratospheric ozone climatology and variability over a southern subtropical site: Reunion Island $\left(21^{\circ} \mathrm{S} ; 55^{\circ} \mathrm{E}\right)$. Annales of Geophysics, v. 25, p. 2321-2334, 2007.

SOLOMON, S. Stratospheric ozone depletion: a review of concepts and history. Rev.Geophy., v. 37, n. 3, p. 275-316, 1999.
TANG, Q.; PRATHER, M. J. Correlating tropospheric column ozone with tropopause folds: the AuraOMI satellite data. Atmos. Chem. Phys. Discuss., v. 10, p. 14875-14896, 2010.

TRICKL, T.; B ̈̈RTSCH-RITTER, N.; EISELE, H.; FURGER, M.; MÜCKE, R.; SPRENGER, M.; STOHL, A. High-ozone layers in the middle and upper troposphere above Central Europe: potential import from the stratosphere along the subtropical jet stream. Atmos. Chem. Phys., v. 11, p. 9343-9366, 2011.

WAUGH, D. W. Subtropical stratospheric mixing linked to disturbances in the polar vortices. Nature, v. 365, p. 535-537, 1993.

WAUGH, D.; PLUMB, R.; ATKINSON, R. J.; SCHOEBERL, M. R.; LAIT, L. R.; NEWMAN, P. A.; LOEWENSTEIN, M.; TOOHET, D.; AVALLONE, L.; WEBSTER, C.; MAY, R. Transport out of the lower stratospheric vortex by Rossby wave breaking. J. Geophys. Res-Atmos., v. 99, p. 1071-1088, 1994.

VAN LOON, H. Blocking action in Southern Hemisphere. Part 1. Notos, v. 5, p.171-175, 1956. 